\title{
Erratum to: Early response evaluation for recurrent high grade gliomas treated with bevacizumab: a volumetric analysis using diffusion-weighted imaging
}

\author{
Eui Jin Hwang • Yongjun Cha • A. Leum Lee - Tae Jin Yun - Tae Min Kim • \\ Chul-Kee Park · Ji-Hoon Kim · Chul-Ho Sohn · Sung-Hye Park • \\ Il Han Kim • Dae Seog Heo $\cdot$ Se-Hoon Lee $\cdot$ Seung Hong Choi
}

Published online: 14 March 2013

(C) Springer Science+Business Media New York 2013

\section{Erratum to: J Neurooncol \\ DOI 10.1007/s11060-013-1072-z}

In the legend included in the artwork of Fig. $3 b$ of the original publication, the interval specifications for the two curves were incorrect. They should have read ' $\leq 50 \%$ ' and '>50\%' for the blue and red curves, resp. (just as they did in the caption to the figure).

\section{S.-H. Park}

Department of Pathology, Seoul National University College of Medicine, 28, Yongon-dong, Chongno-gu, Seoul 110-744,

South Korea

\section{H. Kim}

Department of Radiation Oncology, Cancer Research Institute, Seoul National University College of Medicine, 28,

Yongon-dong, Chongno-gu, Seoul 110-744, South Korea

Yongon-dong, Chongno-gu, Seoul 110-744, South Korea

e-mail: shlee119@snu.ac.kr

C.-K. Park

Department of Neurosurgery, Seoul National University College of Medicine, 28, Yongon-dong, Chongno-gu, Seoul 110-744,

South Korea
S. H. Choi $(\square)$

Center for Nanoparticle Research, Institute for Basic Science, and School of Chemical and Biological Engineering,

Seoul National University, Seoul 151-742, Korea e-mail: verocay@snuh.org 